\title{
Automatic-box (CVT) without hydraulics
}

\author{
Konstantin Ivanov ${ }^{1,2, *}$ \\ ${ }^{1}$ Chair “Control Systems for Aerospace Engineering”, Almaty University of Power Engineering and Telecommunications, Almaty, \\ Kazakhstan \\ ${ }^{2}$ Laboratory of Adaptive Mechanisms, Institute of Mechanics and Machine Science MON of Republic Kazakhstan, Almaty, Kazakhstan
}

Email address:
ivanovgreek@mail.ru

\section{To cite this article:}

Konstantin Ivanov. Automatic-Box (CVT) without Hydraulics. American Journal of Mechanics and Applications. Special Issue: Adaptive Transmissions. Vol. 2, No. 6-1, 2014, pp. 13-20. doi: 10.11648/j.ajma.s.2014020601.13

\begin{abstract}
Mechanical automatic-box is developed on the basis of discovery «Effect of force adaptation in mechanics». Automatic- box represents the gear differential with two degrees of freedom which has a cogwheels closed contour. Closed contour imposes additional constraint on motion of links and provides definability of motion. Automatic-box has constant engagement of cogwheels. Transfer ratio of the box is independently brought into accord to output loading. Laws of transfer parameters interaction in geometrical and analytical form are presented in the work.
\end{abstract}

Keywords: Automatic-Box, Gear Differential, Two Degrees of Freedom, Closed Contour

\section{Introduction}

Any saw the automatic-box. It is the most complex aggregate in the car after the engine. The aggregate contains next subassemblies: hydraulic converter stepped gearing, switching mechanism. And box cost approach to engine cost The aggregate uses a complex control system and demands thin adjustment. Nevertheless it supposes jerks and failures. It would be very good for the driver if here to use a gear wheels with smoothly varying transfer ratio. Smoothness of motion can be reached in transfer with constant engagement of cogwheels without switched stages. «Such gearing does not exist» - you will tell. However - the fantastic fact! Gearing with constant engagement of wheels and with variable transfer ratio exists. At first it has appeared in patents of inventors and then the classical theory (without use a friction) has been developed for it on the basis of mechanics laws. It is possible to open the site $<$ http://www.adaptation.kz/> and to see by the eyes this transfer and its work into transfer case. The fantasy variable target loading is forcing to rotate a target shaft with demanded speed at constant power of engine. There is a simply redistribution of motion speeds of links at constant engagement of cogwheels at operating regime.

\section{Comparison of Transfers}

The stepless adjustable transfer (continuously variable transmission - CVT) which is used now contains the hydro transformer and the multistage switched gear planetary mechanism. The hydro transformer carries out additional constraint between links of the planetary mechanism and provides transfer self-regulation. Transfer is complex and bulky. The control system of transfer creates a gear change and does not provide continuous conformity of shaft rotation speed to loading on it.

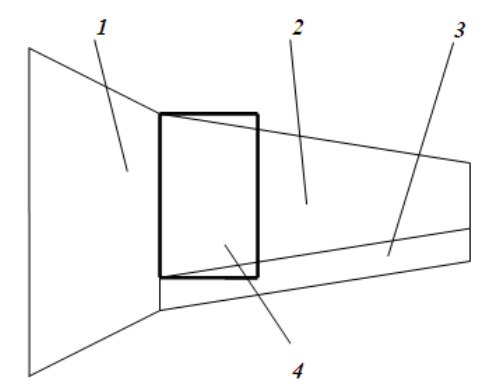

Figure 1. Comparison of hydro mechanical and adaptive-mechanical transmission

Offered gear stepless adjustable transfer is adaptive mechanical transfer. The adaptive mechanical transfer contains only the adaptive gear mechanism and it is extremely simple on a design.

On Fig. 1 hydro mechanical transfer is shown by thin lines. It contains following parts: 1. Hydro transformer. 2. Stepped gearing with 4 or 5 steps of cogwheels. 3. Hydro mechanical switching mechanism.

The adaptive-mechanical transfer which does not have analogues in world practice is shown by thick lines. It 
contains only the adaptive gear mechanism 4 (without hydraulics).

\section{Theoretical Substantiation}

Now it is concerning the transfer theory.

All mechanisms which now are used in the technician for movement transfer from engine to working body have the constant transfer ratio which defines speed of movement of working body. The transmission has some switched gear mechanisms and each mechanism has the transfer ratio. The mechanism with one degree of freedom is kinematical definable - at the given input angular speed of engine the output angular speed of target shaft is strictly defined.

If mechanism with two degrees of freedom to use then it would be possible to operate the transfer ratio and smoothly to change output angular speed. For example, such control has V-belt drive in which diameters of pulleys vary on the move. In this mechanism the second degree of freedom realises change of diameters of pulleys. In the gear mechanism it is impossible to change diameters of cogwheels and number of teeth at constant engagement of wheels. However the gear mechanism with two degrees of freedom in the technician exists. It is gear differential. The gear differential with two degrees of freedom is used in the car to transfer the movement to everyone driving wheel with demanded speed on conditions of movement (for example, on turn). Definability of movement of differential with two degrees of freedom is provided with conditions of car movement (for example, by forces of coupling of driving wheels on trajectories with different radiuses). Thus if the mechanism has two degrees of freedom it can adapt to external conditions in the form of movement resistance forces which impose an additional constraint on this movement.

Theoretical researches have shown that the differential mechanism with two degrees of freedom can be used for movement transfer on one working body (instead of on two). In this case the definiteness of movement is reached also by presence of additional constraint in the form of output moment of resistance on output shaft. Such additional constraint takes place only when differential cogwheels form the closed contour. The differential with the closed contour containing cogwheels represents a transmission with smoothly changing transfer ratio.

\section{Paradox of Mechanisms Theory}

Definability of a kinematic chain with two degrees of freedom having only one input is paradox of mechanisms and machines theory.

According to mechanisms and machines theory [1] the number of degrees of freedom of the mechanism should be equal to number of input links. However, recently patents and publications on adaptive mechanism in the form of two-mobile kinematic chain with the closed contour have been made. Possibility of definability of two-mobile kinematic chain with one input makes paradox of mechanisms and machines theory.

\section{History of Creation}

At first the possibility of practical use of the mechanism with two degrees of freedom and one input has been presented in the patent on nonreaction engine [2] in 1980.

Idea of creation stepless adjustable transfer with constant engagement of cogwheels in the form of hydrodynamic converter and gear differential is presented in patents of $S$. Crockett [3] and I. Wolkov [4]. The mechanism with two degrees of freedom and one input in the form of gear differential is presented in D. Harries's patent [5]. Various variants of designs of gear adaptive transfer in the form of gear differential with two degrees of freedom are presented in K. Ivanov's patents [6 - 9].

For the first time the proof of definability of two-mobile mechanisms with one input has been presented by K. Ivanov in 1995 [10]. Proof of definability of two mobile mechanisms on principle of virtual works was made in $\mathrm{K}$. Ivanov's works [11-13]. Additional constraint between links of two-mobile mechanism if it has the closed contour has been theoretically proved in works [14 - 15]. The theory of adaptive mechanisms has received the further development in works [16, 17, 18, 19 and 20].

There are different ways of the proof of kinematic definability of gear differential with two degrees of freedom [11 - 17]. Visual way of proof based on considering of picture of speeds $[16,17]$ is used in present work. Geometrical image of additional constraint is presented and the proof of existence of additional constraint causing paradox of the mechanisms and machines theory is brought.

The research problem is a development of theoretical laws of automatic-box parameters interaction for creation of actual designs on the given working conditions. Work is executed on basis of laws of mechanics and mechanisms and machines theory.

\section{Research of Motion of Gear Differential with Two Degrees of Freedom by Means of Picture of Speeds}

Investigated kinematic chain looks like gear differential with two degrees of freedom (Fig. 2).

Differential contains frame 0 , carrier $H_{1}$, satellite 2 , block of central wheels 1-4, block of ring wheels 3-6, satellite 5 and carrier $\mathrm{H}_{2}$. Cogwheels form for-links closed contour 1-2-3-6-5-4. Sizes of cogwheels 1, 2, 3, 4, 5, 6 are defined by corresponding radiuses $r_{i} \quad i=1,2,3,4,5,6$.

Radiuses of carriers are: $r_{H 1}=r_{1}+r_{2}, r_{H 2}=r_{4}+r_{5}$.

On Figure 3 picture of speeds of the mechanism links $V_{i} \quad i=1,2,3,4,5,6$ is presented. Linear speeds $V_{i}$ 
are expressed via angular speeds $\omega_{i}$ under the formula $V_{i}=\omega_{i} r_{i}$. Linear speeds of carriers are: $V_{H i}=\omega_{H i} r_{H i}, i=1,2$. The initial picture of speeds is shown by full lines. The intermediate picture of speeds is shown by dashed lines. Carrier $H_{1}$ angular speed is defined by line $A b$. Satellite 2 angular speed is presented by the inclined line which is passing through point $b$ matching to carrier $H_{1}$ point $B$. Satellite 5 angular speed is presented by the inclined line which is passing through point $k$ matching to a carrier $H_{2}$ point $K$. Intermediate picture of speeds is presented by dashed lines.

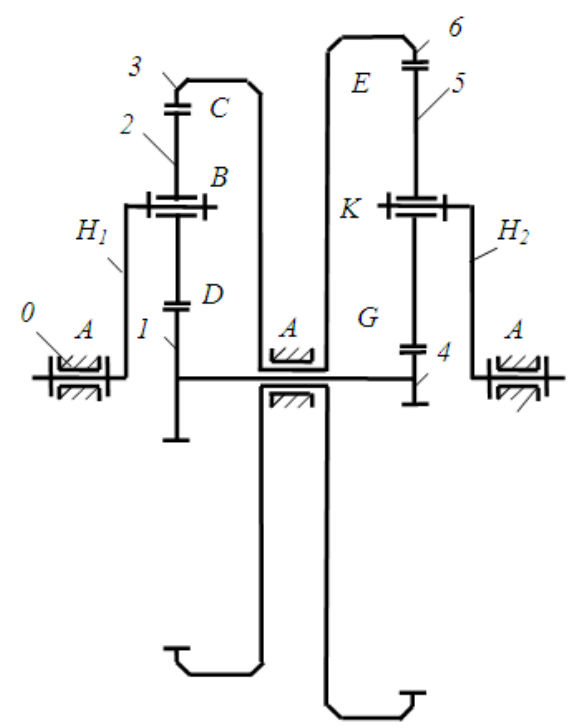

Figure 2. Gear differential

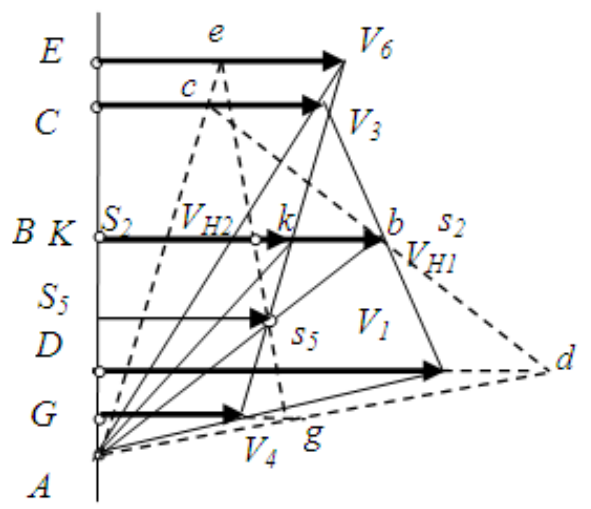

Figure 3. Picture of speeds of the gear differential

Intersection point $S_{5}$ of satellite 5 angular speed line $e g$ with carrier $H_{1}$ angular speed line $A b$ is instantaneous center of rotation (or turn) of satellite 5 concerning carrier $H_{1}$. We will prove that satellite 5 instant centre $S_{5}$ will have constant position on line $A b$ of carrier $H_{1}$ angular speed.

Theorem. In the differential gear mechanism containing two carriers, two satellites and two blocks of central wheels the satellite has constant instantaneous center of rotation on opposite carrier.

For the demonstration at first we will consider kinematic chain picture of speeds in inverse motion at the motionless carrier $H_{1}$ when $V_{H 1}=0$ (Fig. 4).

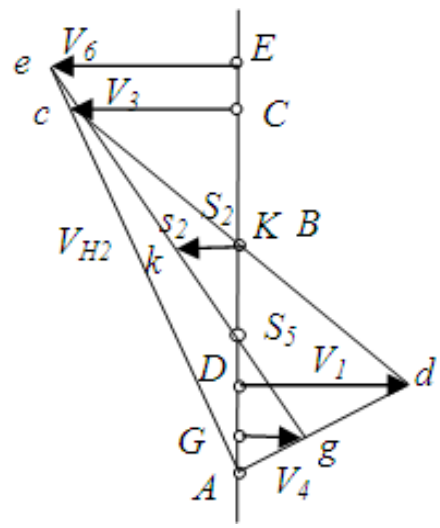

Figure 4. Picture of speeds of the mechanism in inverse motion at motionless input carrier

Linear speed is $V_{H 1}=0$ at motionless carrier $H_{1}$. The satellite 2 instant center of rotation $S_{2}$ coincides with a point $B$. We will run a line $c S_{2} d$ of satellite 2 angular speed. We will trace a line $A c$ of angular speed of link 3-6 and found on it point $e$ defining speed $V_{6}$ of mechanism point $E$. We will trace a line $A d$ of link 1-4 angular speed and found on it a point $g$ defining speed $V_{4}$ of mechanism point $G$. We will trace a line eg of satellite 5 angular speed. Point $S_{5}$ of intersection line eg with a vertical line of zero speeds is the instant center of rotation of the satellite 5.

Intersection point $S_{5}$ of satellite 5 angular speed line $e g$ with vertical line of zero speeds is the instantaneous center of rotation (or turn) of satellite 5 concerning motionless carrier $H_{1}$. The point $S_{5}$ position (size $y=B S_{5}$ ) can be univocal determined through mechanism geometric parameters.

Let's define a point $S_{5}$ position (size $y=B S_{5}$ ) using similitude of triangles.

The sequence of formulation of the equations matches to sequence of geometrical constructions of picture of speeds on Fig. 4.

1) $\frac{C c}{E e}=\frac{r_{3}}{r_{6}}, \frac{D d}{G g}=\frac{r_{1}}{r_{4}}$. As $C c=D d$ then

$$
\frac{E e}{G g}=\frac{r_{1} r_{6}}{r_{3} r_{4}} .
$$

2) $\frac{K k}{E e}=\frac{y}{r_{5}+y}, \frac{K k}{G g}=\frac{y}{r_{5}-y}$. From here

$$
\frac{E e}{G g}=\frac{r_{5}+y}{r_{5}-y} .
$$


From (1) and (2) it follows $\frac{r_{1} r_{6}}{r_{3} r_{4}}=\frac{r_{5}+y}{r_{5}-y}$. From here

$$
y=r_{5} \frac{r_{1} r_{6}-r_{3} r_{4}}{r_{1} r_{6}+r_{3} r_{4}} .
$$

Eq. (3) is univocal determines position of instantaneous center of turn of satellite 5 concerning motionless carrier $H_{1}$. In the valid motion of the mechanism the point $S_{5}$ will move together with the carrier $H_{1}$ as point $s_{5}$ as it is shown in picture of speeds of the moving mechanism (Figure 3). Hence satellite has constant instantaneous centre of rotation on the opposite carrier quot erat demonstrandum.

The invariable position of instant centre $s_{5}$ on carrier

$H_{1}$ defines an additional geometric constraint which takes place in the kinematic chain with two degree of freedom. However this additional constraint has no constructive performance. Mechanically the mechanism remains the kinematic chain with two degree of freedom and keeps the properties.

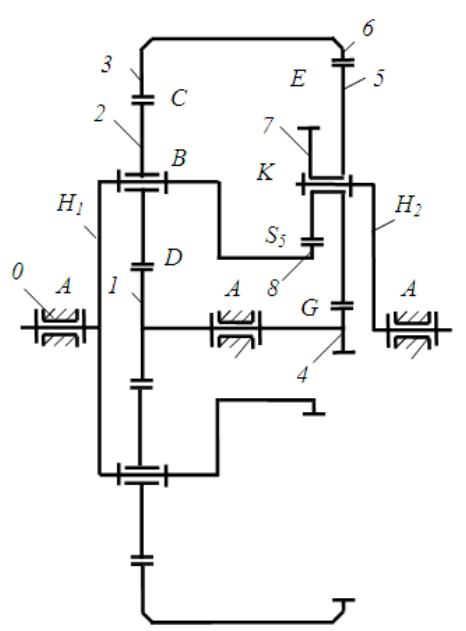

Figure 5. Conditional replacing mechanism with one degree of freedom

Eq. (3) expresses analytically this additional geometric constraint. As a result the kinematic chain becomes definable at presence only one input link. It means that at the set angular speed of the input carrier it is possible to determine speeds of all other mechanism links and points.

The found regularity allows building the conditional replacing mechanism with one degree of freedom (Fig. 5). In the replacing mechanism output satellite is executed in the form of block of wheels 5-7 and affiliated by the higher kinematic pair coinciding with the instant centre $S_{5}$ to the gear ring 8 on input carrier $H_{1}$.

The conditional mechanism will move as a single whole without relative mobility of wheels in the closed contour. However the picture of speeds (Figure 3) admits relative motion of links for the replacing mechanism. Hence constructive additional constraint in the form of cogging of toothed wheels 7,8 of conditional replacing mechanisms is passive.

\section{Force Analysis of Kinematic Chain with Two Degrees Of Freedom}

Considered kinematic chain with two degree of freedom contains two links, having generalized co-ordinates (carriers $H_{1}$ and $H_{2}$ ) and closed contour affiliated to them containing toothed wheels 1-2-3-6-5-4 having zero mobility.

Statement of problem: two generalized forces (the moments of forces $M_{H 1}, M_{H 2}$ on carriers $H_{1}$ and $H_{2}$ ) are given. It is necessary to determine reactions in kinematic pairs.

Solution.

1) Next reaction are transferred from carriers and $\mathrm{H}_{2}$ on satellites 2 and 5 of closed contour

$$
\begin{aligned}
& R_{H 1}=M_{H 1} / r_{H 1}, \\
R_{H 2}= & M_{H 2} / r_{H 2} .
\end{aligned}
$$

2) Reaction $R_{H 1}$ in point $B$ and reactions $R_{12}=R_{32}=R_{H 1} / 2=M_{H 1} / 2 r_{H 1}$ in points $D$ and $C$ act on satellite 2 .

Condition of satellite 2 equilibrium is represented in the form of equality to null of the moments concerning the instant centre of speeds $S_{20}$ (Fig. 4)

$$
R_{12} \cdot D S_{20}+R_{32} \cdot C S_{20}=R_{H 1} \cdot B S_{20}
$$

3) It is analogous reaction $R_{H 2}$ in point $K$ and reactions $R_{45}=R_{65}=R_{H 2} / 2=M_{H 2} / 2 r_{H 2}$ in points $G$ and $E$ act on the satellite 5. Condition of equilibrium of satellite 5 is presented in the form of equality to null of moments concerning the motionless instant centre of speeds $S_{50}$ (it is not shown at Fig. 4)

$$
R_{45} \cdot G S_{50}+R_{65} \cdot E S_{50}=R_{H 2} \cdot K S_{50}
$$

4) We will multiplication Eq. (6) on $\omega_{2}$. With the account $\omega_{2} \cdot D S_{20}=V_{1}, \quad \omega_{2} \cdot C S_{20}=V_{3}, \quad \omega_{2} \cdot B S_{20}=V_{H 1} \quad$ we will gain

$$
R_{12} \cdot V_{1}+R_{32} \cdot V_{3}=R_{H 1} \cdot V_{H 1}
$$

Let's multiplication Eq. (7) on $\omega_{5}$. With the account $\omega_{5} \cdot G S_{50}=V_{4}, \quad \omega_{5} \cdot E S_{50}=V_{6}, \quad \omega_{5} \cdot K S_{50}=V_{H 2} \quad$ we will gain

$$
R_{45} \cdot V_{4}+R_{65} \cdot V_{6}=R_{H 2} \cdot V_{H 2}
$$

Let's add Eq. (8) and Eq. (9)

$$
R_{12} \cdot V_{1}+R_{32} \cdot V_{3}+R_{45} \cdot V_{4}+R_{65} \cdot V_{6}=R_{H 1} \cdot V_{H 1}+R_{H 2} \cdot V_{H 2}
$$

Eq. (9) contains the forces acting on the closed contour from toothed wheels 1-2-3-6-5-4 affiliated to two carriers (generalized co-ordinates). The left side of Eq. (9) represents the sum of powers (or works) of internal forces of the closed 
contour. The right side of Eq. (9) represents the sum of powers (or works) external forces of the closed contour. We will consider kinematic pairs of the closed contour ideal. Then

$$
R_{12} \cdot V_{1}+R_{32} \cdot V_{3}+R_{45} \cdot V_{4}+R_{65} \cdot V_{6}=0
$$

From Eq. (9) follows

$$
R_{H 1} \cdot V_{H 1}+R_{H 2} \cdot V_{H 2}=0
$$

Taking into account expressions (4) we will gain

$$
M_{H 1} \cdot \omega_{H 1}+M_{H 2} \cdot \omega_{H 2}=0
$$

From Eq. (12) follows that one of the generalized forces should be negative. It is necessary to consider, for example that $M_{H 2}$ is moment of resistance on output carrier $\mathrm{H}_{2}$ and $M_{H 1}$ is driving moment on input carrier $H_{1}$. Thus it will appear that in the kinematic chain with two degree of freedom and with the closed contour only one link (input carrier $H_{1}$ ) can have generalized co-ordinate (independent angular speed $\left.\omega_{H 1}\right)$. Such kinematic chain will have the kinematic definability as Eq. (12) allows at the set moments $M_{H 1}$ and $M_{H 2}$ to determine output angular speed $\omega_{H 2}$.

Hence the kinematic chain with two degrees of freedom and with the closed contour at presence only one entry will have static and kinematic definability and will be the mechanism.

It is necessary to note: Eq. (12) cannot be fair for any kinematic chain with two degrees of freedom and one input. From Eq. (12) follows

$$
\omega_{H 2}=\frac{M_{H 1} \cdot \omega_{H 1}}{M_{H 2}} .
$$

Eq. (13) expresses effect of power adaptation. At the set constant parameters $M_{H 1}$ and $\omega_{H 1}$ of input power the output angular speed $\omega_{H 2}$ inversely proportional to set variable output moment of resistance $M_{H 2}$. We will name the mechanism creating effect of power adaptation as the adaptive mechanism.

\section{Transition of Kinematic Chain from Condition with One Degree of Freedom in Operation Regime of Work}

At the start the output carrier $\mathrm{H}_{2}$ is motionless (Fig. 2). The system is in a condition with one degree of freedom.

Let's consider regularity of system transition in condition with two degree of freedom. We will proceed from assumption that output moment of resistance $M_{H 2}$ on output carrier $\mathrm{H}_{2}$ is equal in beginning of motion to input driving moment $M_{H 1}$ on input carrier $H_{1}$. Then according to Eq. (13) angular speeds of input and output carriers will appear equal. Kinematic chain will be rotate round central axis as a single whole in a condition with one degree of freedom (without relative mobility of wheels in the closed contour).

Transition in a condition with two degree of freedom will begin at performance of condition $M_{H 2}>M_{H 1}$. The increase in output moment of resistance in the kinematic chain with two degree of freedom will lead not to a stop of output carrier but to relative motion of wheels in the closed contour. The kinematic chain gets an additional geometric constraint which provides kinematic and static definability.

\section{Automatic-Box Test}

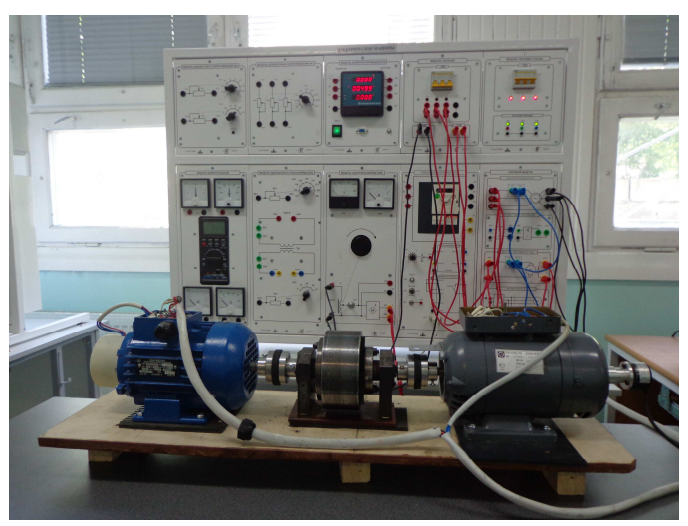

Figure 6. Stand for automatic-box test

Stock-taking of the force adaptation effect in the mechanism made under the scheme presented on Fig. 2 has been executed on the test bed (Fig. 6).

At the stand the electric motor is placed at the left, the electric generator simulating useful target loading, is placed on the right, adaptive-mechanical transfer is placed in the centre between the electric motor and the electric generator.

Theoretical results will be co-ordinated with results of tests at the stand. Presence of force adaptation effect defines a discovery in mechanics area.

\section{The Discovery Formula}

The force adaptation effect in the mechanics consists that the kinematic chain with two degrees of freedom containing input link, output link and the closed mobile contour placed between them provides motion of output link with speed inversely proportional to loading on it at constant input power.

\section{Scientific and Practical Value of Discovery}

The developed discovery allows providing the variable transfer ratio only at the expense of use of the closed contour and its properties without application of the control means 
changing structure of the mechanism. Mechanical properties of the closed contour allow to provide the demanded transfer ratio independently, stepless and automatically.

Creation gear stepless adjustable transfer in the form of the gear closed differential mechanism with two degrees of freedom is theoretically proved.

It is proved that the mobile closed mechanical contour of transfer creates additional constraint and provides the transitive mode of motion translating the mechanism of transfer from an one-mobile condition at start-up into two-mobile condition of an operational mode of motion. It is proved that in an operational mode of motion the equilibrium by a principle of virtual works providing a stepless transfer regulation takes place.

The found laws allow synthesizing gear stepless adjustable transfer on the given operational parameters of motion to execute the kinematic and dynamic analysis of transfer and to define transfer design data.

Gear stepless adjustable transfer in the form of the gear closed differential mechanism with constant engagement of wheels is the elementary transfer of such type and has a reliability corresponding to reliability of the gear mechanism. The specified properties allow using transfer both in easy local drives of manipulators and in heavy drives of transport machines [19] including in motors-wheels [20].

The gear closed differential mechanism of transfer possesses effect of force adaptation to variable technological loading. Force adaptation allows creating the easy and hard loaded adaptive drives of machines with the variable transfer ratio depending on technological resistance (bicycle, motorcycle, car, chisel installation, bulldozer, lorry, etc.).

\section{Practical Implementation}

Practical realization includes the following:

1. The patent of Germany № 202012101273.1.

The patent of Russia № 2398989.

Patents of Kazakhstan: №3208, №11042, №12236, №14477，№17378，№ 023907，№24181，№24625，№ 26107.

2. On Fig. 7 the assembly drawing of adaptive mechanical transfer for conveyor drive is presented.

3. Computer animation model of the automatic-box is presented on a site http:www.adaptation.kz. It shows a motion of links at change of input loading. The animation model contains the engine (grey color at the left), transfer without the case (the input carrier with input satellite of green color, block of solar wheels of red color, block of ring wheels of dark blue color, output satellite of green color with output carrier and brake (on the right of red color) simulating output resistance moment. The engine has constant parameters of power (angular speed and driving moment). The brake creates the variable resistance moment. At increase of output resistance moment the output carrier slows down motion and on the contrary. Key W increases the output moment of resistance. Key Q reduces the output moment of resistance. Keys with arrows change model position.

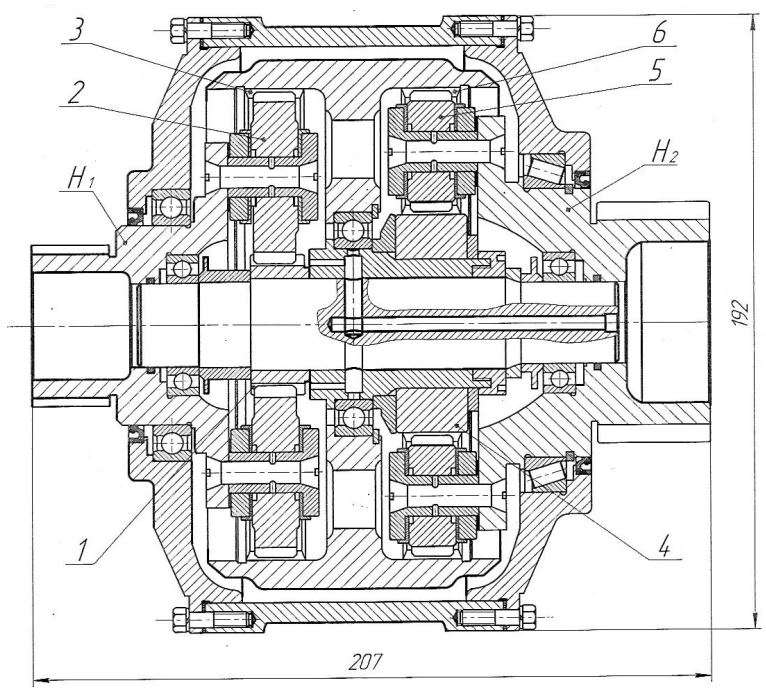

Figure 7. Assembly drawing of automatic-box

5. Computer model of adaptive mechanical transfer (Fig. $8)$.

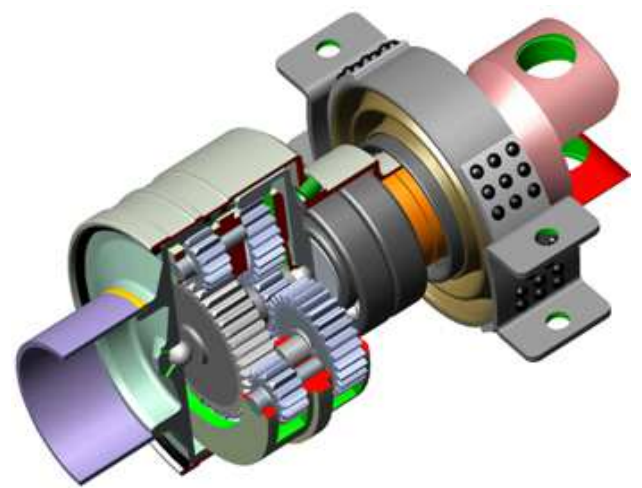

Figure 8.. Computer model of adaptive mechanical transfer

\section{Discussions on the Problem}

Professor S.U.Dzholdasbekov (Institute of Mechanics and Machine Sciece of Kazakhstan): Hydrodynamic transfer is also actually transfer with two degrees of freedom. The transfer ratio of hydrodynamic transfer can change from 1 to the maximum value (nearby 2 ) when there is an output shaft stop. At the moment of output shaft stop efficiency is equal 0 . Mechanical adaptive transfer works in a similar range of transfer ratios. Therefore mechanical adaptive transfer will have analogous efficiency.

K.S.Ivanov: In hydrodynamic transfer the target moment is created by an internal hydraulic moment of resistance which depends on a difference of angular speeds of pump and turbine wheels. The kinematic pair of pump and turbine wheels cannot be an ideal kinematic pair. This pair functionally exists for transformation of an internal moment of resistance to an external output resistance moment. The hydraulic moment of resistance simultaneously defines factor of an energy loss. Therefore efficiency of 
hydrodynamic transfer linearly decreases depending on loading.

Basic other dependence occurs in mechanical adaptive transfer. This dependence is defined by the formula (13), connecting the external moments and speeds. The internal mechanical moment in the closed contour of mechanical adaptive transfer also occurs. However this moment does not create energy loss, as power (or work) internal forces is equal to null. In mechanical adaptive transfer there is only a disproportionate of speeds of motion of links in the closed contour.

Thus efficiency of mechanical adaptive transfer functionally does not depend on external loading and matches to efficiency of the planet gear. Efficiency instantly gets a zero value at the moment of qualitative change of the mechanism structure at stop of output link.

\section{Conclusion}

The automatic- box represents the gear differential with two degrees of freedom which has a cogwheels closed contour. The gear differential with two degrees of freedom and one input containing the closed contour has the adaptation to output loading and definability of motion.

Definability of a kinematic chain with two degrees of freedom having only one input is paradox of mechanisms and machines theory.

It is proved that gear differential with two degrees of freedom containing closed contour has additional geometrical constraint which provides definability of motion in the presence of one input. Paradoxical additional constraint takes place in a picture of speeds in the form of instantaneous relative centre of rotation of satellite and carrier. This additional constraint can be presented structurally as passive superfluous constraint. Paradoxical constraint analytically connects power and kinematic parameters of an input and an output. This additional analytical constraint is expression of force adaptation effect which provides in the mechanism overcoming of variable output loading at constant input power. Mechanical properties of the closed contour allow to provide the demanded transfer ratio independently, stepless and automatically without use of hydraulics and application of the control means changing geometrical parameters of the mechanism.

The box-automatic in the form of gear differential with constant engagement of cogwheels is a dream of designers and inventors. It has fantastic advantages: extreme simplicity of a design, small sizes, high efficiency and full automatic adjustable. It is not surprising, that till now nobody accepts new idea seriously.

\section{References}

[1] Levitsky N.I. Theory of mechanisms and machines. M, Science. 1979. $576 \mathrm{p}$.
[2] Ivanov K.S., Dmitrieva N.A. Without reaction engine. Copyright certificate of USSR №769157 from 7.09.1980. 8 p.

[3] Samuel J. Crockett. Shiftless, continuously-aligning transmission. Patent of USA 4,932,928, Cl. F16H 47/08, U.S. Cl. 475/51; 475/47.1990, 9 p.

[4] Wolkov I.V. Way of automatic and continuous change of the twisting moment and speed of rotation of a target shaft depending on resistance to motion and the device for its realisation. Invention description to the patent of Russia RU 2234626 from 27.03.2004. 16 p.

[5] Harries John. Power transmission system comprising two sets of epicyclic gears. Patent of Great Britain GB2238090 (A). 1991, $11 \mathrm{p}$.

[6] Ivanov K.S. Transfer with automatically adjustable speed. Preliminary patent of republic Kazakhstan № 3208 from 15.03.1996. $12 \mathrm{p}$.

[7] Ivanov K.S. Adaptive tooth gearing (variants). Preliminary patent RK №14477 from 13.04.2004. 9 p.

[8] Ivanov K.S., Yaroslavtsev E.K. Way of automatic and continuous change of the twisting moment and speed of rotation of a target shaft depending on resistance to motion and the device for its realisation. Patent of Russia RU № 2398989. 10.09.2010. $10 \mathrm{p}$.

[9] Ivanov Konstantin S., Almaty, KAZ - Owner of the registered sample. The name - Device of automatic and continuous change of a twisting moment - and changes of a corrected speed of output shaft depending on a tractive resistance. The deed on registration of the registered sample № 202012101 273.1. Day of Registration 02.05.2012. German patent and firm establishment. Federal Republic Germany. 2012. 12 p.

[10] Ivanov K.S. The Question of the Synthesis of Mechanical Automatic Variable Speed Drives. Proceedings of the Ninth World Congress on the Theory of Machines and Mechanisms, Vol. 1, Politechnico di Milano, Italy, August 29-Sept 2, 1995. P. $580-584$.

[11] Ivanov K.S. Discovery of the Force Adaptation Effect. Proceedings of the 11th World Congress on Mechanism and Machine Science V. 2. April 1 - 4, 2004, Tianjin, China. P. $581-585$.

[12] Ivanov K.S. Gear Automatic Adaptive Variator with Constant Engagement of Gears. Proceedings of the 12th World Congress in Mechanism and Machine Science. Besancon. France. 2007, Vol. 2. P. 182 - 188.

[13] Ivanov K.S. The simplest automatic transfer box. WCE 2010. World Congress on Engineering 2010 (ICME) London, UK. 2010. P. $1179-1184$.

[14] Ivanov K.S. Effect of force adaptation in mechanics. Journal of Mechanics Engineering and Automation. Vol. 1, N 3. Libertiville, USA. 2011. P. $163-180$.

[15] Ivanov K.S. Toothed continuously variable transmission (CVT) for transport. EngOpt 2012 - 3rd International Conference on Engineering Optimization. Rio de Janeiro, Brazil, 01 - 05 July 2012. P. 211 - 218.

[16] Ivanov K.S. Paradox of theory of mechanisms and machines. Modern mechanical engineering. Science and education. Materials of 3rd International scientifically-practical conference. St.-Petersburg state polytechnical university. Union of machine engineers of Russia. St.-Petersburg. Russia. 2013. P. 146 - 156. 
[17] Ivanov K.S. Paradox in the Mechanism Science. 1-st International Symposium on the Education in Mechanism and Machine Science. Madrid. Spain. 2013. P. 132-138.

[18] Ivanov K.S. Paradox of mechanics - a basis of creation CVT. Transactions of 2-d IFToMM Asian Conference on Mechanisms and Machines Science. November 7-10, 2012, Tokyo, Japan. P. $245-264$.
[19] Ivanov K.S. Drive of Extreme Transport Technique. Applied Mechanics and Materials. Vol. 245 (2013), Trans Tech. Publications, Swizerland. 2013. P 185-190.

[20] Ivanov K.S. Self-Adjusting Motor-Wheel with CVT. International Journal of Engineering and Innovanive Technology (IJEIT). Volume 2, Issue 4. Florida. USA. 2012. P.189- 195 . 